\title{
THE COEFFICIENTS IN AN ASYMPTOTIC EXPANSION ${ }^{1}$
}

L. CARLITZ

Put

$$
e^{n z}=\sum_{r=0}^{n} \frac{(n z)^{r}}{r !}+\frac{(n z)^{n}}{n !} S_{n}(z),
$$

where $n$ is a positive integer and $z$ an arbitrary complex number. Ramanujan [4, p. 26] asserted (in a different notation) that

$$
S_{n}(1)=\frac{n !}{2}\left(\frac{e}{n}\right)^{n}-\frac{2}{3}+\frac{4}{135 n}+O\left(\frac{1}{n^{2}}\right) .
$$

Copson [2] proved that $\left\{S_{n}(-1)\right\}$ is a decreasing sequence with limit $-1 / 2$ and derived an asymptotic series. In a recent paper, Buckholtz [1] proved that, for $k \geqq 1$,

$$
S_{n}(z)=\sum_{r=0}^{k-1}\left(\frac{1}{n}\right)^{r} U_{r}(z)+O\left(n^{-k}\right)
$$

uniformly in a certain region of the z-plane. The coefficients $U_{r}(z)$ are determined by

$$
U_{r}(z)=(-1)^{r}\left(\frac{z}{1-z} \frac{d}{d z}\right)^{r} \frac{z}{1-z} .
$$

It follows from (1) that

$$
U_{r}(z)=(-1)^{r} \frac{Q_{r}(z)}{(1-z)^{2 r+1}},
$$

where, for $r \geqq 1, Q_{r}(z)$ is a polynomial of degree $r$ with positive integral coefficients.

To find an explicit expression for $U_{r}(z)$, we put

$$
F=F(z, t)=\sum_{k=0}^{\infty} U_{k}(z) t^{k} / k ! .
$$

Then, by (1),

Received by the editors October 3, 1963.

1 This work was supported in part by NSF Grant GP-1593. 


$$
\left(\frac{z}{1-z} \frac{\partial}{\partial z}\right) F=-\sum_{k=0}^{\infty} U_{k+1}(z) t^{k} / k !=-\frac{\partial F}{\partial t}
$$

so that

$$
\frac{z}{1-z} \frac{\partial F}{\partial z}+\frac{\partial F}{\partial t}=0
$$

The system

$$
\frac{1-z}{z} d z=d t=\frac{d F}{0}
$$

has the particular integrals

$$
F, \quad z e^{-z-t} \text {. }
$$

Hence (4) has the solution

$$
F=\phi\left(z e^{-z-t}\right)
$$

where $\phi$ is arbitrary. Since

$$
F(z, 0)=\frac{z}{1-z}
$$

it is evident that

$$
\phi\left(z e^{-z}\right)=\frac{z}{1-z} .
$$

Now it is known [3, p. 126, no. 214] that

$$
\frac{e^{\alpha z}}{1-z}=\sum_{n=0}^{\infty} \frac{(n+\alpha)^{n}}{n !}\left(z e^{-z}\right)^{n} \text {. }
$$

It follows that

$$
\frac{z}{1-z}=\sum_{n=1}^{\infty} \frac{n^{n}}{n !}\left(z e^{-z}\right)^{n} .
$$

Comparing (7) with (6) it is clear that $\phi$ is determined. Thus (5) becomes

$$
F(z, t)=\sum_{n=1}^{\infty} \frac{n^{n}}{n !}\left(z e^{-s-t}\right)^{n}
$$

We have therefore 


$$
\begin{aligned}
F(z, t) & =\sum_{r=1}^{\infty} \frac{r^{r}}{r !}\left(z e^{-z}\right)^{r} \sum_{k=0}^{\infty}(-1)^{r} \frac{r^{k} t^{k}}{k !} \\
& =\sum_{k=0}^{\infty}(-1)^{k} \frac{t^{k}}{k !} \sum_{r=1}^{\infty} \frac{r^{r+k}}{r !}\left(z e^{-z}\right)^{r},
\end{aligned}
$$

so that

$$
\begin{aligned}
U_{k}(z) & =(-1)^{k} \sum_{r=1}^{\infty} \frac{r^{r+k}}{r !}\left(z e^{-z}\right)^{r} \\
& =(-1)^{k} \sum_{r=1}^{\infty} \frac{r^{r+k}}{r !} z^{r} \sum_{s=0}^{\infty}(-1)^{s} \frac{r^{s} z^{s}}{s !} \\
& =(-1)^{k} \sum_{n=1}^{\infty} \frac{z^{n}}{n !} \sum_{r=1}^{n}(-1)^{n-r}\left(\begin{array}{l}
n \\
r
\end{array}\right) r^{n+k}
\end{aligned}
$$

We put

(9)

$$
S(n+k, n)=\frac{1}{n !} \sum_{r=0}^{n}(-1)^{n-r}\left(\begin{array}{l}
n \\
r
\end{array}\right) r^{n+k}
$$

so that $S(n+k, n)$ is a Stirling number of the second kind [5, p. 33]. Thus

$$
U_{k}(z)=(-1)^{k} \sum_{n=1}^{\infty} z^{n} S(n+k, n)
$$

for all $k \geqq 0$.

It follows from (2) and (10) that

$$
Q_{k}(z)=(1-z)^{2 k+1} \sum_{n=1}^{\infty} z^{n} S(n+k, n) .
$$

If we put

$$
Q_{k}(z)=\sum_{n=1}^{k} a_{k n} z^{n} \quad(k \geqq 1),
$$

it is clear that

$$
a_{k n}=\sum_{j=0}^{n}(-1)^{j}\left(\begin{array}{c}
2 k+1 \\
j
\end{array}\right) S(n-j+k, n-j) .
$$

For example, since 
$S(k, 1)=1, \quad S(k, 2)=\frac{1}{2 !}\left(2^{k}-2\right), \quad S(k, 3)=\frac{1}{3 !}\left(3^{k}-3 \cdot 2^{k}+3\right)$,

we find that

$$
\begin{aligned}
a_{k 1} & =1 \quad(k \geqq 1), \\
a_{k 2} & =S(2+k, 2)-(2 k+1) S(1+k, 1) \\
& =\frac{1}{2}\left(2^{k+2}-2\right)-(2 k+1) \\
& =2^{k+1}-2(k+1), \\
a_{k 3} & =S(3+k, 3)-(2 k+1) S(2+k, 2)+\left(\begin{array}{c}
2 k+1 \\
2
\end{array}\right) S(1+k, 1) \\
& =\frac{1}{6}\left(3^{3+k}-3 \cdot 2^{3+k}+3\right)-(2 k+1)\left(2^{k+1}-1\right)+\left(\begin{array}{c}
2 k+1 \\
2
\end{array}\right) \\
& =\frac{1}{2}\left(3^{2+k}+1\right)-(2 k+3) 2^{k+1}+(2 k+1)(k+1) .
\end{aligned}
$$

In particular we have

$$
Q_{1}(z)=z, \quad Q_{2}(z)=z+2 z^{2}, \quad Q_{3}(z)=z+8 z^{2}+6 z^{3}
$$

in agreement with Buckholtz.

It follows from (1) and (2) that

$$
Q_{k+1}(z)=(2 k+1) z Q_{k}(z)-z(z-1) Q_{k}^{\prime}(z) .
$$

Combining (13) with (11) we get the recurrence

$$
a_{k n}=n a_{k-1, n}+(2 k-n) a_{k-1, n-1},
$$

from which it is clear that the $a_{k n}$ are positive integers for $1 \leqq n \leqq k$. By means of (14) we can easily compute the following table.

$$
\begin{array}{rrrrrrr}
1 & & & & & \\
1 & 2 & & & & \\
1 & 8 & 6 & & & \\
1 & 22 & 58 & 24 & & \\
1 & 52 & 328 & 444 & 120 & \\
1 & 114 & 1452 & 4400 & 3708 & 720
\end{array}
$$

As a check we note that

$$
Q_{k}(1)=\sum_{n=1}^{k} a_{k n}=1 \cdot 3 \cdot 5 \cdots(2 k-1) ;
$$

this is an immediate consequence of (13). 


\section{REFERENCES}

1. J. D. Buckholtz, Concerning an approximation of Copson, Proc. Amer. Math. Soc. 14 (1963), 564-568.

2. E. T. Copson, An approximation connected with $e^{-3}$, Proc. Edinburgh Math. Soc. (2) 3 (1932/1933), 201-206.

3. G. Polya and G. Szegö, Aufgaben und Lehrsätze aus der Analysis. I, Springer, Berlin, 1925.

4. S. Ramanujan, Collected papers, Cambridge Univ. Press, Cambridge, 1927.

5. J. Riordan, An introduction to combinatorial analysis, Wiley, New York, 1958.

DUKE UNIVERSITY

\section{ON CLASSES OF UNIVALENT CONTINUED FRACTIONS}

T. L. HAYDEN ${ }^{1}$ AND E. P. MERKES ${ }^{2}$

1. Introduction. From results of Leighton and Scott [3], there is a unique one-to-one correspondence between formal power series $w^{-1}+\sum_{n=2}^{\infty} c_{n} w^{-n}$ and $C$-fractions

$$
F(w)=\frac{1}{w}-\frac{a_{1}}{w^{\delta_{1}}}-\frac{a_{2}}{w^{\delta_{2}}}-\cdots-\frac{a_{n}}{w^{\delta_{n}}}-\cdots,
$$

where $\delta_{n}$ is an integer, $\delta_{1} \geqq 0, \delta_{n+1}+\delta_{n} \geqq 1$, and $a_{n+p}=0$ whenever $a_{p}=0$ for $n=1,2, \ldots$. For a fixed continued fraction (1.1), let $K_{F}$ denote the class of formal power series which correspond to $C$-fractions of the form

$$
\frac{1}{w}-\frac{a_{1}^{\prime}}{w^{\delta_{1}}}-\frac{a_{2}^{\prime}}{w^{\delta_{2}}}-\cdots-\frac{a_{n}{ }^{\prime}}{w^{\delta_{n}}}-\cdots,
$$

where $\left|a_{n}^{\prime}\right| \leqq\left|a_{n}\right|, n=1,2, \cdots$. In order that each power series in $K_{F}$ represent an analytic function in $|w| \geqq 1$ it is necessary and sufficient that $\left|a_{n}\right| \leqq g_{n}\left(1-g_{n-1}\right)$, where $0<g_{n-1} \leqq 1, n=1,2, \cdots$, and $g_{p-1}=1$ if and only if $a_{p}=0[2$, p. 374]. Conditions on the parameters $g_{n}$ of the chain sequence $\left\{g_{n}\left(1-g_{n-1}\right)\right\}_{n=1}^{\infty}$ which imply that each

Received by the editors October 16, 1963.

${ }^{1}$ Sponsored by the Mathematics Research Center, U. S. Army, Madison, Wisconsin under Contract No. DA-11-022-ORD-2059. 然

2 Sponsored in part by the Mathematics Research Center, U. S. Army, Madison, Wisconsin under Contract No. DA-11-022-ORD-2059 and in part by the Air Force Office of Scientific Research. 